\title{
Análise Descritiva do Perfil dos Exames Citopatológicos do Colo do Útero Realizados em Mulheres Indígenas e Não Indígenas no Brasil, 2008-2011
}

Descriptive Analysis of the Profile of Cytopathologic Cervical Exams Carried out in Indigenous and Non-Indigenous Women in Brazil, 2008-2011 Análisis Descriptiva del Perfil de la Citopatología del Cuello del Útero Realizada en las Mujeres Indígenas y No Indígenas en Brasil, 2008-2011

\author{
Maria Asunción Solé Pla ; Flávia Miranda Corrêa ${ }^{2}$; Itamar Bento Claro ${ }^{3}$ Marcos André Felix da Silva ${ }^{4}$; Maria Beatriz Kneipp Dias ${ }^{5}$; \\ Paula Chagas Bortolon ${ }^{6}$
}

\section{Resumo}

Introduçáo: Existem no Brasil 408.056 mulheres indígenas. Essa população é mais vulnerável aos agravos de grande magnitude, entre eles o câncer do colo do útero. Contudo existem poucos estudos sobre essa questão entre indígenas. Objetivo: Descrever o perfil dos exames citopatológicos realizados na população indígena brasileira em comparação a não indígenas. Método: Análise descritiva de frequência e proporção dos exames citopatológicos realizados entre mulheres indígenas e não indígenas registradas no Sistema de Informação do Câncer do Colo do Útero (SISCOLO) segundo regiōes de residência para o período de 2008 a 2011. Resultados: Entre 2008 e 2010, 8\% dos exames citopatológicos realizados no país registraram a informação de cor/raça, sendo que em 2011 esta proporção aumentou para 12,4\%. Em indígenas e não indígenas, respectivamente, foram realizados 11.964 e 3.738 .707 exames satisfatórios; foram considerados insatisfatórios $1 \%$ e 1,2\% dos exames; 74,9\% e 77,2\% dos exames foram realizados na faixa etária de 25 a 64 anos; $20 \%$ e $17,5 \%$ dos exames foram realizados abaixo dos 25 anos; 83,4\% e 76,5\% dos exames foram realizados no intervalo de 1 a 2 anos; $2,1 \%$ e 2,9\% dos exames apresentaram-se alterados. A razão lesão de alto grau / câncer invasor do colo do útero passou de 11,5, em 2009, para 16,1, em 2011, em não indígenas e de 1,7 para 5,0 entre indígenas. Conclusáo: Os dados evidenciam a importância do tema para as mulheres indígenas, sugerem dificuldade de acesso aos exames citopatológicos para esse grupo, e ressaltam a necessidade de melhoria da informação cor/raça no SISCOLO.

Palavras-chave: Neoplasias do Colo do Útero; População Indígena; Sistemas de Informação; Esfregaço Vaginal

\footnotetext{
${ }^{1}$ Médica. Mestre em Saúde Coletiva. Tecnologista da Divisão de Detecção Precoce e Apoio à Organização de Rede. Coordenação-Geral de Prevenção e Vigilância (Conprev). Instituto Nacional do Câncer José de Alencar Gomes da Silva (INCA). Ministério da Saúde (MS). Rio de Janeiro (RJ), Brasil. E-mail: masuncion@inca.gov.br.

${ }^{2}$ Médica. Doutoranda e Mestre em Saúde da Criança e da Mulher. Analista da Divisão de Detecção Precoce e Apoio à Organização de Rede/Conprev/ INCA/MS. Rio de Janeiro (RJ), Brasil. E-mail: fcorrea@inca.gov.br.

${ }_{3}^{3}$ Assistente Social. Mestre em Políticas Públicas de Saúde. Tecnologista da Divisão de Detecçáo Precoce e Apoio à Organização de Rede/Conprev/INCA/ MS. Rio de Janeiro (RJ), Brasil. E-mail: iclaro@inca.gov.br.

${ }^{4}$ Enfermeiro. Mestre em Ensino de Ciências da Saúde e do Ambiente. Tecnologista da Divisão de Detecção Precoce e Apoio à Organização de Rede/ Conprev/INCA/MS. Rio de Janeiro (RJ), Brasil.E-mail: mfelix@inca.gov.br.

${ }^{5}$ Enfermeira. Mestre em Epidemiologia. Tecnologista da Divisão de Detecçấo Precoce e Apoio à Organização de Rede/Conprev/INCA/MS. Rio de Janeiro (RJ), Brasil.E-mail: mdias@inca.gov.br.

${ }^{6}$ Farmacêutica. Mestre em Ciências da Saúde pela Escola Nacional de Saúde Pública Sérgio Arouca (ENSP). Fundaçăo Oswaldo Cruz (Fiocruz)/MS. Prestadora de Serviço Técnico Especializado para os Programas Nacionais de Controle de Câncer. Divisão de Detecção Precoce e Apoio à Organização de Rede/Conprev/INCA/MS. Rio de Janeiro (RJ), Brasil.E-mail: pbortolon@inca.gov.br.

Endereço para correspondência: Maria Asunción Solé Pla. Rua Marquês do Pombal, 125 - 7o andar - Centro. Rio de Janeiro (RJ), Brasil. CEP: $20230-240$.
} 


\section{INTRODUÇÃO}

Estudos internacionais apontam o câncer do colo do útero como um importante problema de saúde pública entre as populaçóes indígenas ${ }^{1-3}$.

Segundo dados do Censo do Instituto Brasileiro de Geografia e Estatística (IBGE) de $2010^{4}$, existem no país 408.056 mulheres indígenas. A Política Nacional de Atenção à Saúde dos Povos Indígenas chama atenção para a maior vulnerabilidade da populaçáo indígena aos agravos de grande magnitude na população brasileira, entre eles, o câncer do colo do útero ${ }^{5}$. O relatório de gestão da Fundação Nacional de Saúde e Departamento de Saúde Indígena de 2010 afirma que o controle desse câncer nas mulheres indígenas é uma prioridade e que foram realizados com este fim 86.360 exames citopatológicos no período de 2003 a 2008 e 18.818 em 2009 (dados ainda parciais $)^{6}$.

O Ministério da Saúde (MS) destaca a importância da realizaçáo de açôes direcionadas para a prevenção e tratamento do câncer do colo do útero que levem em conta o contexto cultural dos povos indígenas e que produzam dados epidemiológicos suficientes para a avaliação de açóes desenvolvidas para este grupo ${ }^{7}$. Apesar disto, no Brasil, são escassos os dados sobre a saúde dos povos indígenas principalmente sobre esse câncer ${ }^{8-11}$.

Indígenas estáo mais expostas a fatores de risco para infecçáo pelo HPV como início precoce da atividade sexual, multiparidade, múltiplos parceiros sexuais, prevalência de Doenças Sexualmente Transmissíveis e a outros fatores associados ao desenvolvimento do câncer do colo do útero como a imunodeficiência e o tabagismo ${ }^{8-12}$. Entretanto, não foram encontrados dados estatísticos ou evidências científicas que confirmem uma maior incidência e mortalidade por essa causa em mulheres indígenas em comparação às não indígenas no país.

O exame citopatológico ou exame Papanicolaou é internacionalmente reconhecido como uma técnica de rastreio eficiente para a redução da mortalidade pelo câncer do colo do útero ${ }^{13}$. Ele faz parte da linha de cuidado do programa de controle desse câncer no país, juntamente com a confirmação diagnóstica, seguimento ou tratamento das lesôes precursoras ou invasoras eventualmente encontradas ${ }^{14}$.

No Brasil, o Sistema de Informação do Câncer do Colo do Útero (SISCOLO) é o sistema informatizado de cadastro dos exames citopatológicos e histopatológicos no âmbito do SUS ${ }^{15}$. Sáo registrados no SISCOLO cerca de 10 milhóes de exames citopatológicos por ano no país ${ }^{16}$. As informaçóes de cor e raça autorreferidas passaram a constar nesse sistema a partir de 2008, possibilitando descrever o perfil dos exames citopatológicos realizados na população indígena brasileira em comparação a não indígena, objetivo deste estudo.

A identificação de possíveis diferenças e especificidades entre os grupos estudados pode orientar açóes mais adequadas propiciando melhores resultados do Programa Nacional de Controle do Câncer do Colo do Útero.

\section{MÉTODO}

Foi realizada uma análise descritiva dos exames citopatológicos do colo do útero constantes no SISCOLO no período de 2008 a 2011, entre mulheres indígenas e não indígenas. O SISCOLO é o sistema informatizado desenvolvido em 1998 pelo Instituto Nacional do Câncer José de Alencar Gomes da Silva (INCA) em parceria com o Departamento de Informática do Sistema Único de Saúde (DATASUS) para gerenciamento de informaçôes provenientes do programa de controle do câncer do colo do útero. Implementado através da Portaria SAS/ MS no 408/99, constituiu-se em um subsistema do Sistema de Informação Ambulatorial do Sistema Único de Saúde (SIA/SUS). Além de cadastrar os exames citopatológicos e histopatológicos no âmbito do SUS, o sistema permite, entre outros, registrar informaçóes sobre condutas diagnósticas e terapêuticas relativas aos exames positivos ou alterados e a coletar dados para construção de indicadores ${ }^{15}$. Trata-se, portanto, de uma importante fonte de informaçóes desse câncer.

Foram consideradas não indígenas as brancas, pretas, pardas e amarelas. Os dados foram acessados por meio do TabNet, aplicativo de consulta on-line desenvolvido pelo DATASUS. Os dados referentes ao ano de 2011, apesar de parciais, foram incluídos nas análises devido ao quantitativo de exames em mulheres indígenas naquele ano.

Para o estudo, foram analisadas completude da informação, a proporção de exames em indígenas, grau de insatisfatoriedade, distribuição de exames por faixa etária, periodicidade e tempo de realização do último exame, resultado de exames e a razão lesão de alto grau/ carcinoma invasor.

A completude da informação cor/raça no sistema foi avaliada através da proporção de seu preenchimento segundo ano e regiáo de residência. A verificação da consistência deste dado buscou averiguar a qualidade de preenchimento dessa variável no SISCOLO.

Entre os exames com raça/cor informada, foi calculada a proporção de exames realizados em mulheres indígenas segundo ano e região de residência.

As proporçóes de exames insatisfatórios entre mulheres indígenas e não indígenas segundo regiāo de residência foram comparadas, para o período estudado. Segundo a Nomenclatura Brasileira para Laudos Cervicais ${ }^{17}$, 
são insatisfatórias as amostras, colhidas para exame citopatológico, que apresentem materiais acelulares ou hipocelulares ou que tenham leitura prejudicada por grande presença de sangue, piócitos ou outros contaminantes que impossibilitem sua leitura. Esses exames são descartados e uma nova coleta deve ser realizada. Para as análises subsequentes, incluíram-se apenas os exames considerados satisfatórios.

A distribuição etária das mulheres indígenas e não indígenas que realizaram os exames no período foi comparada segundo regiōes de residência.

A proporção de existência de citologia anterior relatada foi descrita para o grupo etário de 25 a 64 anos. A partir dessa informação, a periodicidade de realização dos exames foi avaliada comparando-se entre indígenas e não indígenas e segundo regiốes, a proporção de exames para os quais o exame anterior havia sido realizado há um ou dois anos.

Os resultados dos exames citopatológicos foram apresentados por meio de proporções considerando a frequência de cada categoria estudada entre o total de exames, para regiáo, grupo etário e as categorias de raça/ cor. Foram considerados exames "alterados" ou positivos aqueles com resultados compatíveis com atipias de significado indeterminado em células escamosas ou glandulares possivelmente não neoplásicas ou quando não se pode excluir lesão intraepitelial de alto grau, lesôes intraepiteliais de baixo e alto graus, carcinoma epidermoide invasor, adenocarcinoma in situ e adenocarcinoma invasor. Dentro da classificação "lesóes intraepiteliais de alto grau", foram incluídas as "lesóes intraepiteliais de alto grau em que não se pode excluir microinvasão".

Ademais, a razão lesão de alto grau /carcinoma invasor foi usada como um indicador de diagnóstico oportuno versus tardio do câncer do colo do útero, uma vez que se espera, com um bom programa de detecção precoce, que haja o diagnóstico de um maior número de lesōes precursoras (de alto grau) em comparação ao número de casos de câncer.

\section{RESULTADOS}

\section{INFORMAÇ̃ES DE COR E RAÇA NO SISCOLO}

Cerca de $8 \%$ dos exames citopatológicos realizados no país entre 2008 e 2010 registraram a informação de cor/raça. No ano de 2011, essa proporção aumentou para 12,4\%. Entre as regiōes do país, em 2011, as Regióes Centro-Oeste e Norte foram as que apresentaram melhores proporçōes de preenchimento, respectivamente $24,4 \%$ e $18,1 \%$.

Entre os exames com informação cor/raça conhecida no período estudado, $59,9 \%$ eram brancas, $37,1 \%$ negras ou pardas, $2,7 \%$ amarelas e $0,3 \%$ indígenas.

\section{EXAMES CITOPATOLÓGICOS ENTRE MULHERES INDÍGENAS}

No período de 2008 a 2011, constam no SISCOLO informaçôes sobre 11.964 exames citopatológicos satisfatórios realizados em mulheres indígenas e 3.738.707 em mulheres não indígenas. Foram considerados insatisfatórios $1 \%$ dos exames realizados em mulheres indígenas e $1,2 \%$ nas não indígenas. A variação entre regiōes não apresentou diferenças consideráveis.

Desde o início da inclusão da informação cor/raça no SISCOLO, o número de exames citopatológicos realizados em mulheres indígenas vem crescendo. Em 2008, apenas 1.358 (0,2\%) exames traziam essa informação. Em 2011, este número chegou a 6.226 , representando $0,6 \%$ do total. Os maiores percentuais de exames em mulheres indígenas ocorreram nas Regióes Centro-Oeste e Nordeste como apresentado na Tabela 1 .

A distribuição etária das mulheres indígenas examinadas mostra que, para o período do estudo, 74,9\% dos exames

Tabela 1.Exames Citopatológicos realizados em mulheres indígenas segundo ano e região de residência. Brasil, 2008 a 2011

\begin{tabular}{|c|c|c|c|c|c|c|c|c|c|c|c|c|c|c|c|}
\hline \multirow{2}{*}{ Região } & \multicolumn{3}{|c|}{2008} & \multicolumn{3}{|c|}{2009} & \multicolumn{3}{|c|}{2010} & \multicolumn{3}{|c|}{2011} & \multicolumn{3}{|c|}{ Total período } \\
\hline & total & indígena & $\%$ & total & indígena & $\%$ & total & indígena & $\%$ & total & indígena & $\%$ & total & indígena & $\%$ \\
\hline Norte & 51.999 & 100 & 0,2 & 43.689 & 116 & 0,3 & 47.994 & 26 & 0,1 & 93.761 & 166 & 0,2 & 237.443 & 408 & 0,2 \\
\hline Nordeste & 283.884 & 307 & 0,1 & 341.725 & 535 & 0,2 & 276.671 & 266 & 0,1 & 201.238 & 2.663 & 1,3 & 1.103 .518 & 3.771 & 0,3 \\
\hline Sudeste & 408.077 & 838 & 0,2 & 365.982 & 1.121 & 0,3 & 432.966 & 1.678 & 0,4 & 557.504 & 212 & 0,0 & 1.764 .529 & 3.849 & 0,2 \\
\hline Sul & 72.411 & 66 & 0,1 & 108.210 & 61 & 0,1 & 82.023 & 123 & 0,1 & 103.880 & 120 & 0,1 & 366.524 & 370 & 0,1 \\
\hline Centro-Oeste & 26.538 & 47 & 0,2 & 43.407 & 221 & 0,5 & 34.404 & 233 & 0,7 & 174.308 & 3.065 & 1,8 & 278.657 & 3.566 & 1,3 \\
\hline Total & 842.909 & 1.358 & 0,2 & 903.013 & 2.054 & 0,2 & 874.058 & 2.326 & 0,3 & 1.130 .691 & 6.226 & 0,6 & 3.750 .671 & 11.964 & 0,3 \\
\hline
\end{tabular}

Excluídos casos com informação ignorada

"Total" se refere aos exames realizados em todas as mulheres, indígenas e não indígenas

"Indígena" se refere apenas aos exames realizados em mulheres indígenas

Fonte: Siscolo. Acesso em 15/02/2012 
foram realizados na faixa etária de 25 a 64 anos, definida como alvo do programa de controle do câncer do colo do útero pelas diretrizes nacionais ${ }^{11}$. Mulheres menores de 20 anos realizaram 7,8\% dos exames e aquelas de 20 a 24 anos, 12,2\%. Entre o total de exames de cada região, a Norte apresentou maior índice $(30,9 \%)$ de citologia entre mulheres menores de 25 anos. Na Região Centro-Oeste, esse percentual foi de $26,1 \%$.

Para as não indígenas, $77,2 \%$ dos exames foram realizados na faixa etária de 25 a 64 anos, 6,4\% em menores de 20 anos e $11,1 \%$ em mulheres de 20 a 24 anos. Nas Regiốes Norte e Nordeste, 20,2\% e 19,7\% foram registrados em menores de 25 anos respectivamente (Tabela 2).

Verificou-se, no país, de 2008 a 2011, aumento na proporção de realização de citologia anterior, tanto em mulheres indígenas quanto nas não indígenas. Em 2008, entre as de 25 a 64 anos de idade, esse percentual foi de 65,7\% para as indígenas e 80,9\% para as não indígenas. Em 2011, essas proporçôes passaram para 81,5\% e 86,5\%, respectivamente.

Quanto à periodicidade de exames, a maioria apresenta periodicidade menor que a recomendada pelas Diretrizes Brasileiras ${ }^{14}$, sendo que, em 2011, 83,4\% dos exames em indígenas de 25 a 64 anos de idade havia referência do último exame ter sido realizado há um ou dois anos. Entre as não indígenas, esse percentual foi de 76,5\% (Tabela 3).

Quanto aos resultados dos exames (Tabela 4), no período estudado, 2,1\% exames citopatológicos de mulheres indígenas e $2,9 \%$ nas não indígenas apresentaram-se alterados. Tiveram diagnóstico de lesão de baixo grau, $0,48 \%$ dos exames em indígenas e $0,87 \%$ em não indígenas. Lesóes de alto grau foram identificadas em $0,33 \%$ do primeiro grupo e $0,32 \%$ do segundo. Carcinoma invasor foi diagnosticado em $0,08 \%$ dos exames em indígenas e $0,02 \%$ em não indígenas. A proporção de adenocarcinoma in situ e invasor foi de $0,01 \%$ para as indígenas e $0,02 \%$ para náo indígenas.

As Regiôes Norte e Centro-Oeste apresentaram as maiores proporçôes de exames alterados $(5,4 \%$ e $4 \%)$, de lesôes de baixo grau $(0,74 \%$ e $1,09 \%)$ e alto grau $(0,49 \%$ e $0,64 \%)$ entre os exames de mulheres indígenas. Já entre as não indígenas, as maiores proporçóes observaram-se nas Regiốes Sudeste e Centro-Oeste.

Entre os nove resultados de carcinoma invasor entre indígenas, quatro residiam na Região Nordeste, quatro na Centro-Oeste e um na Região Norte. A proporção de carcinoma invasor na Região Norte foi a maior, com $0,25 \%$ do total de exames dessa Região.

Tabela 2. Exames citopatológicos segundo grupo etário e região de residência entre indígenas e não indígenas. Brasil, 2008 a 2011

\begin{tabular}{|c|c|c|c|c|c|c|c|c|c|c|c|c|c|}
\hline \multirow{2}{*}{ Região e faixa etária } & \multicolumn{2}{|c|}{ Até 11 anos } & \multicolumn{2}{|c|}{12 a 14 anos } & \multicolumn{2}{|c|}{15 a 19 anos } & \multicolumn{2}{|c|}{20 a 24 anos } & \multicolumn{2}{|c|}{25 a 64 anos } & \multicolumn{2}{|c|}{65 e mais } & \multirow{2}{*}{$\begin{array}{c}\begin{array}{c}\text { Total } \\
\text { exames }\end{array} \\
\mathbf{n}^{\mathbf{0}}\end{array}$} \\
\hline & $n^{0}$ & $\%$ & $n^{0}$ & $\%$ & $n^{0}$ & $\%$ & $n^{0}$ & $\%$ & $n^{0}$ & $\%$ & $n^{0}$ & $\%$ & \\
\hline \multicolumn{14}{|l|}{ Norte } \\
\hline indígenas & 1 & 0,2 & 13 & 3,2 & 46 & 11,3 & 66 & 16,2 & 258 & 63,2 & 24 & 5,9 & 408 \\
\hline não indígenas & 158 & 0,1 & 1.065 & 0,4 & 15.732 & 6,6 & 30.987 & 13,1 & 182.440 & 77,0 & 6.653 & 2,8 & 237.035 \\
\hline \multicolumn{14}{|l|}{ Nordeste } \\
\hline indígenas & 7 & 0,2 & 13 & 0,3 & 182 & 4,8 & 454 & 12,0 & 2916 & 77,3 & 199 & 5,3 & 3771 \\
\hline não indígenas & 2.741 & 0,2 & 6.804 & 0,6 & 68.712 & 6,2 & 138.265 & 12,6 & 833.076 & 75,8 & 50.149 & 4,6 & 1.099 .747 \\
\hline \multicolumn{14}{|l|}{ Sudeste } \\
\hline indígenas & 8 & 0,2 & 21 & 0,5 & 218 & 5,7 & 344 & 8,9 & 2969 & 77,1 & 289 & 7,5 & 3849 \\
\hline não indígenas & 761 & 0,0 & 5.117 & 0,3 & 97.561 & 5,5 & 179.232 & 10,2 & 1.368 .879 & 77,7 & 109.130 & 6,2 & 1.760 .680 \\
\hline \multicolumn{14}{|l|}{ Sul } \\
\hline indígenas & 0 & 0,0 & 4 & 1,1 & 32 & 8,6 & 44 & 11,9 & 275 & 74,3 & 15 & 4,1 & 370 \\
\hline não indígenas & 301 & 0,1 & 945 & 0,3 & 21.081 & 5,8 & 35.117 & 9,6 & 288.167 & 78,7 & 20.543 & 5,6 & 366.154 \\
\hline \multicolumn{14}{|l|}{ Centro-0este } \\
\hline indígenas & 0 & 0,0 & 39 & 1,1 & 345 & 9,7 & 546 & 15,3 & 2.542 & 71,3 & 94 & 2,6 & 3.566 \\
\hline não indígenas & 159 & 0,1 & 1.051 & 0,4 & 17.348 & 6,3 & 30.622 & 11,1 & 213.271 & 77,5 & 12.640 & 4,6 & 275.091 \\
\hline \multicolumn{14}{|l|}{ Total } \\
\hline indígenas & 16 & 0,1 & 90 & 0,8 & 823 & 6,9 & 1.454 & 12,2 & 8.959 & 74,9 & 621 & 5,2 & 11.964 \\
\hline não indígenas & 4.120 & 0,1 & 14.982 & 0,4 & 220.434 & 5,9 & 414.223 & 11,1 & 2.885 .833 & 77,2 & 199.115 & 5,3 & 3.738 .707 \\
\hline
\end{tabular}

Excluídos casos com informaçấo ignorada

Fonte: Siscolo. Acesso em 15/02/2012 
Tabela 3. Proporções de exames com informação de citologia anterior e de realização de último preventivo há um ou dois anos em indígenas e não indígenas com 25 a 64 anos de idade. Brasil, 2008 e 2011

\begin{tabular}{l|c|c|c|c|c|c}
\hline \multirow{2}{*}{\begin{tabular}{c}
\multirow{2}{*}{$\begin{array}{c}\text { Região } \\
\text { de residência }\end{array}$} \\
\cline { 2 - 7 }
\end{tabular}} & \multicolumn{2}{|c|}{$\begin{array}{c}\text { \% Exames com citologia anterior } \\
\text { 2008 }\end{array}$} & \multicolumn{2}{c|}{2011} & \multicolumn{2}{c}{2011} \\
\cline { 2 - 7 } & Indígena & $\begin{array}{c}\text { Náltimo } \\
\text { indígena }\end{array}$ & Indígena & $\begin{array}{c}\text { Não } \\
\text { indígena }\end{array}$ & Indígena & Não indígena \\
\hline Norte & 43,8 & 80,9 & 48,9 & 85,3 & 84,9 & 74,0 \\
\hline Nordeste & 57,2 & 74,7 & 77,4 & 81,5 & 82,1 & 77,6 \\
\hline Sudeste & 67,2 & 85,2 & 82,7 & 88,9 & 66,9 & 76,3 \\
\hline Sul & 90,7 & 79,3 & 85,9 & 82,2 & 87,8 & 77,4 \\
\hline Centro-Oeste & 79,2 & 90,1 & 86,8 & 88,3 & 85,3 & 76,4 \\
\hline Total & 65,7 & 80,9 & 81,5 & 86,5 & 83,4 & 76,5 \\
\hline
\end{tabular}

Excluídos casos com informaçáo ignorada

Fonte: Siscolo. Acesso em 15/02/2012

Tabela 4.Resultados de exames citopatológicos segundo região de residência e grupos etários em indígenas e não indígenas. Brasil, 2008 a 2011

\begin{tabular}{|c|c|c|c|c|c|c|c|c|c|c|c|c|c|}
\hline \multirow[t]{2}{*}{ Região e faixa etária } & \multirow{2}{*}{$\begin{array}{c}\text { Total de } \\
\text { exames } \\
n^{\circ}\end{array}$} & \multicolumn{2}{|c|}{$\begin{array}{l}\text { Exames } \\
\text { alterados }\end{array}$} & \multicolumn{2}{|c|}{$\begin{array}{c}\text { Lesão de baixo } \\
\text { grau }\end{array}$} & \multicolumn{2}{|c|}{$\begin{array}{l}\text { Lesão de } \\
\text { alto grau }\end{array}$} & \multicolumn{2}{|c|}{$\begin{array}{l}\text { Carcinoma e. } \\
\text { invasor }\end{array}$} & \multicolumn{2}{|c|}{$\begin{array}{l}\text { Adenocarc. } \\
\text { in situ }\end{array}$} & \multicolumn{2}{|c|}{$\begin{array}{l}\text { Adenocarc. } \\
\text { invasor }\end{array}$} \\
\hline & & $n^{0}$ & $\%$ & $n^{0}$ & $\%$ & $n^{0}$ & $\%$ & $n^{0}$ & $\%$ & $n^{0}$ & $\%$ & $n^{0}$ & $\%$ \\
\hline \multicolumn{14}{|l|}{ Norte } \\
\hline indígenas & 408 & 22 & 5,39 & 3 & 0,74 & 2 & 0,49 & 1 & 0,25 & 0 & 0,0 & 0 & 0,00 \\
\hline não indígenas & 237.035 & 3.840 & 1,62 & 1.718 & 0,72 & 659 & 0,28 & 119 & 0,05 & 7 & 0,00 & 10 & 0,00 \\
\hline \multicolumn{14}{|l|}{ Nordeste } \\
\hline indígenas & 3.771 & 35 & 0,93 & 8 & 0,21 & 10 & 0,27 & 4 & 0,11 & 0 & 0,0 & 0 & 0,00 \\
\hline não indígenas & 1.099 .747 & 19.695 & 1,79 & 6.757 & 0,61 & 2.239 & 0,20 & 265 & 0,02 & 57 & 0,01 & 42 & 0,00 \\
\hline \multicolumn{14}{|l|}{ Sudeste } \\
\hline indígenas & 3.849 & 30 & 0,78 & 5 & 0,13 & 3 & 0,08 & 0 & 0,00 & 0 & 0,0 & 1 & 0,03 \\
\hline não indígenas & 1.760 .680 & 65.857 & 3,74 & 18.100 & 1,03 & 7.166 & 0,41 & 377 & 0,02 & 92 & 0,01 & 138 & 0,01 \\
\hline \multicolumn{14}{|l|}{ Sul } \\
\hline indígenas & 370 & 15 & 4,05 & 2 & 0,54 & 1 & 0,27 & 0 & 0,00 & 0 & 0,0 & 0 & 0,00 \\
\hline não indígenas & 366.154 & 9.153 & 2,50 & 2.601 & 0,71 & 833 & 0,23 & 32 & 0,01 & 13 & 0,00 & 11 & 0,00 \\
\hline \multicolumn{14}{|l|}{ Centro-Oeste } \\
\hline indígenas & 3.566 & 144 & 4,04 & 39 & 1,09 & 23 & 0,64 & 4 & 0,11 & 0 & 0,0 & 0 & 0,00 \\
\hline não indígenas & 275.091 & 9.742 & 3,54 & 3.277 & 1,19 & 1.175 & 0,43 & 108 & 0,04 & 20 & 0,01 & 23 & 0,01 \\
\hline \multicolumn{14}{|l|}{ Menor de 20 anos } \\
\hline indígenas & 929 & 29 & 3,12 & 12 & 1,29 & 0 & 0,00 & 0 & 0,00 & 0 & 0,00 & 0 & 0,00 \\
\hline não indígenas & 239.536 & 10.843 & 4,53 & 5.576 & 2,33 & 366 & 0,15 & 5 & 0,00 & 1 & 0,00 & 2 & 0,00 \\
\hline \multicolumn{14}{|l|}{20 a 24 anos } \\
\hline indígenas & 1.454 & 31 & 2,13 & 14 & 0,96 & 4 & 0,28 & 0 & 0,00 & 0 & 0,00 & 0 & 0,00 \\
\hline não indígenas & 414.223 & 15.049 & 3,63 & 6.566 & 1,59 & 1.062 & 0,26 & 12 & 0,00 & 6 & 0,00 & 5 & 0,00 \\
\hline \multicolumn{14}{|l|}{25 a 64} \\
\hline indígenas & 8.960 & 169 & 1,89 & 29 & 0,32 & 29 & 0,32 & 8 & 0,09 & 0 & 0,00 & 0 & 0,00 \\
\hline não indígenas & 2.885 .833 & 76.990 & 2,67 & 19.736 & 0,68 & 9.771 & 0,34 & 610 & 0,02 & 157 & 0,01 & 159 & 0,01 \\
\hline \multicolumn{14}{|l|}{ Maior de 64} \\
\hline indígenas & 621 & 17 & 2,74 & 2 & 0,32 & 6 & 0,97 & 1 & 0,16 & 0 & 0,00 & 1 & 0,16 \\
\hline não indígenas & 199.115 & 5.405 & 2,71 & 575 & 0,29 & 873 & 0,44 & 274 & 0,14 & 25 & 0,01 & 58 & 0,03 \\
\hline \multicolumn{14}{|l|}{ Total } \\
\hline indígenas & 11.964 & 246 & 2,06 & 57 & 0,48 & 39 & 0,33 & 9 & 0,08 & 0 & 0,0 & 1 & 0,01 \\
\hline não indígenas & 3.738 .707 & 108.287 & 2,90 & 32.453 & 0,87 & 12.072 & 0,32 & 901 & 0,02 & 189 & 0,01 & 224 & 0,01 \\
\hline
\end{tabular}

Excluídos casos com informaçáo ignorada

Fonte: Siscolo. Acesso em 15/02/2012 
Em relação ao grupo etário, a maior proporção de exames alterados, em mulheres indígenas e não indígenas ocorreu em mulheres mais jovens, com menos de 25 anos de idade $(2,52 \%$ e $3,96 \%$ respectivamente). Esse grupo etário também apresentou as maiores proporçôes de lesóes de baixo grau (1,09\% em indígenas e 1,86\% em não indígenas). A proporçáo de lesão de alto grau e de câncer foi maior nos grupos de idade acima de 25 e mais evidente em maiores de 64 anos, em ambos os grupos raciais. Não houve lesáo de alto grau em mulheres indígenas menores de 20 anos de idade e ocorreram dois casos de carcinoma invasor, na faixa de 25 a 29 anos e outro na de 35 a 39 anos.

A Razão Lesão de alto grau / Câncer invasor do colo do útero no país apresentou aumento, passando de 11,5 em 2008 para 16,1 em 2011 em não indígenas. Entre mulheres indígenas, apesar do crescimento, os valores são inferiores, chegando a 5,0 em 2011 (Figura 1).

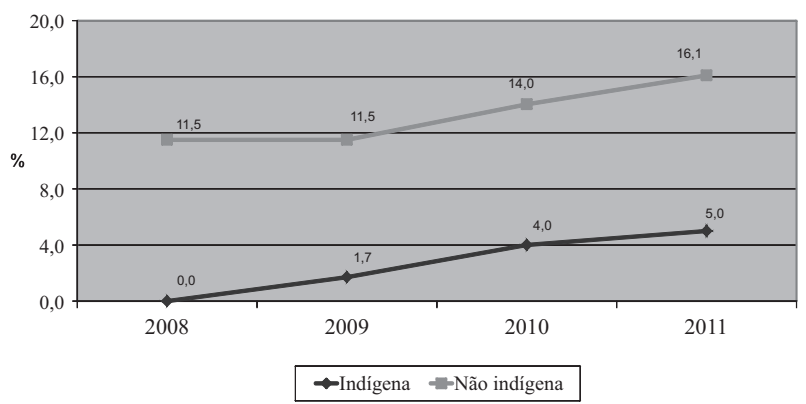

Figura 1. Razão entre lesão de alto grau e câncer invasor do colo do útero em mulheres indígenas e não indígenas. Brasil, 2008 a 2011

\section{DISCUSSÃO}

Apesar de a informação de cor/raça estar disponível no SISCOLO desde 2008, a proporção de seu preenchimento é baixa. O preenchimento desses dados em outros sistemas de informação apresenta-se maior. Em 93\% dos registros do Sistema de Informação de Mortalidade (SIM) em 2009, há informação de cor/raça. No Sistema de Internação Hospitalar (SIH-SUS), 66\% dos registros contêm essa informação em $2011^{18}$. Também o número de exames citopatológicos realizados em indígenas segundo relatório da SESAI é bem maior do que o registrado no SISCOLO ${ }^{6}$.

A representação de mulheres indígenas entre o total de exames constantes no SISCOLO é próxima aos resultados de distribuição cor/raça do censo IBGE de $2010^{4}$, no qual, $0,4 \%$ da população feminina do país é indígena. Entretanto, difere segundo regiôes onde, segundo o censo, nas Regiōes Norte, Nordeste, Sudeste, Sul e Centro-Oeste as mulheres indígenas representam, respectivamente, $1,9 \%, 0,4 \%, 0,1 \%, 0,3 \%$ e $0,9 \%$ da população feminina. É importante também ressaltar que a variável raça/cor é autorreferida e por isto há limites nos resultados relacionados à subjetividade dessa informação.
O estudo não revelou diferenças significativas quanto à proporção de exames insatisfatórios colhidos em mulheres indígenas e não indígenas. Essa proporção serve como indicador de qualidade da coleta desse exame.

As indígenas realizam maior proporção de exames citopatológicos em idades mais jovens do que as não indígenas, principalmente nas Regiốes Norte $\mathrm{e}$ Centro-Oeste. A conduta de iniciar a coleta antes do recomendado pelas diretrizes nacionais parece associada à interpretação de que indígenas iniciariam atividade sexual mais precocemente e estariam mais expostas às doenças sexualmente transmissíveis ${ }^{8-9,12}$. Entretanto, há necessidade de estudos complementares que justifiquem uma abordagem diferenciada nesta populaçáo e avaliem seu custo-efetividade.

Entre mulheres indígenas, é menor a proporção de exames nos quais há referência de realização de uma citologia anterior, mais evidenciado nas Regiôes Norte e Nordeste. Isto pode indicar uma menor captação desse grupo para realização do exame. Esse índice apresentou melhora no período tanto para indígenas quanto para não indígenas, entretanto, a verificação de altos percentuais de Papanicolaou realizados anual ou bianualmente, mais evidente entre as indígenas, sugere que os exames estão sendo dirigidos às mesmas mulheres, enquanto outras não estão sendo captadas. Segundo a PNAD $2008^{19}, 13 \%$ das mulheres brasileiras de 25 a 69 anos de idade informaram nunca terem realizado esse exame.

Embora as recomendaçóes relacionadas à periodicidade trienal tenham respaldo nas diretrizes de programas europeus e norte americanos bem-sucedidos, no Brasil, encontra-se resistência entre mulheres e profissionais de saúde na sua utilização, sendo o exame anual equivocadamente considerado uma prática mais eficaz e segura ${ }^{20}$.

No presente estudo, encontrou-se proporção de exames alterados ou positivos maior na população não indígena. Também foi observada uma maior proporção de lesôes de baixo grau nesse grupo. Não houve diferença significativa entre os dois grupos quanto às lesóes de alto grau, porém evidenciou-se maior proporção de lesóes invasoras entre as indígenas. Tal situação pode indicar a existência de uma maior dificuldade de acesso às açôes de controle do câncer do colo do útero para as indígenas.

A literatura levantada, referida a seguir, aponta para valores maiores que os encontrados neste estudo, em que a proporção de exames alterados em mulheres indígenas variou de $4,0 \%$ a $16,3 \%$. Esse fato pode ser atribuído às condiçốes inerentes ao cenário experimental, em que os estudos são conduzidos de acordo com protocolos que garantem a qualidade dos diagnósticos citopatológicos.

A proporçáo de lesôes de baixo e alto graus também foi inferior ao encontrado na literatura pesquisada. Taborda 
et al. ${ }^{10}$ avaliaram exames de 423 mulheres do parque do Xingu, realizados entre 1989 e 1996, onde 1,2\% apresentaram lesão de baixo grau, 1,7\% lesão de alto grau e $1,2 \%$ carcinomas invasivos.

Mendes ${ }^{11}$ estudou 424 mulheres indígenas de 16 etnias diferentes, no estado do Pará e encontrou 2,1\% de lesóes de baixo grau, 3,3\% de lesôes de alto grau e 1,2\% de carcinomas invasores.

Rodrigues et al. ${ }^{21}$ investigaram exames de 147 indígenas de etnias Suruí e 197 de etnia Cinta Larga, no período de 2002 a 2004. Os resultados mostraram, entre as mulheres Suruí, 3,4\% atipias de significado indeterminado. Entre as de etnia Cinta Larga, 3,6\% de lesôes de baixo grau, 2\% de alto grau e $0,5 \%$ carcinoma invasor.

Pereira et al..$^{22}$ identificaram, entre 2004 e 2006, em Dourados, Mato Grosso do Sul, 5,1\% de exames com lesôes de baixo grau e $0,6 \%$ alto grau entre 989 mulheres da aldeia Jaguapirú e 1,8\% lesóes de baixo grau e 1,1\% de alto grau na aldeia Bororó. Não houve caso de carcinoma invasor.

Pereira ${ }^{9}$ analisou exames citopatológicos realizados entre 2005 e 2006 em indígenas do Xingu, Mato Grosso. Dos 503 exames realizados, 3\% tiveram lesóes de baixo grau, 1,6\% lesôes de alto grau, 0,2\% carcinoma espinocelular e $0,2 \%$ adenocarcinoma.

Quanto à idade das mulheres no momento do diagnóstico das lesóes, o presente estudo evidenciou, tanto em indígenas quanto em não indígenas, uma maior proporção de lesóes de baixo grau em mulheres mais jovens e lesôes de alto grau e de câncer, em grupos etários mais velhos, como o esperado pela história natural da doença. Chama a atenção os percentuais de lesão de alto grau e de câncer em mulheres maiores de 64 anos de idade, principalmente entre indígenas, merecendo uma análise específica.

A baixa razão entre lesóes de alto grau e câncer invasor verificada em mulheres indígenas reforça a hipótese de maior dificuldade no acesso aos exames citopatológicos para esse grupo, gerando falha no diagnóstico oportuno com baixa captação de lesôes precursoras, que são de mais fácil tratamento e melhor prognóstico.

\section{CONCLUSÃO}

O estudo contribuiu para esclarecer a dimensão da questão concernente ao câncer do colo do útero em mulheres indígenas, comparando as condiçóes dessa patologia entre tais mulheres e as demais pertencentes a outros grupos raciais. Seus resultados apontam para a melhoria da oferta dos exames citopatológicos no período estudado, principalmente para o grupo indígena. Nota-se, entretanto, uma tendência de início da coleta antes dos
25 anos e a repetiçáo de exames com periodicidade maior que a preconizada pelas atuais diretrizes clínicas nacionais. Apesar de a frequência de lesóes encontrada ser inferior às referidas nos estudos levantados, os resultados do estudo sugerem o diagnóstico tardio do câncer do colo do útero entre as mulheres indígenas.

É imprescindível considerar que as informaçôes de 2011 sofreram interferência da obrigatoriedade de inclusão, a partir da portaria SAS/MS no 508/2010, da informação etnia para os exames realizados em mulheres de cor/raça indígena. Enquanto alguns estados da federação referiram ter melhorado seu preenchimento, outros relatam perdas em um primeiro momento, como o evidenciado na Região Sudeste. Esse aumento das informaçóes de forma diferenciada nas regiôes do país pode ter gerado um viés na comparação entre os dados da população indígena e não indígena. Ademais, a atual versão do SISCOLO traz dados referentes aos exames realizados e não sobre as mulheres. Por isto, deve-se considerar a possibilidade de duplicidade de exames por uma mesma mulher.

Apesar das restriçóes existentes, o presente trabalho endossa a importância do tema para a saúde da mulher indígena e reforça a necessidade esforços para a melhoria do preenchimento das informaçôes de cor/raça no SISCOLO, que pode ser um importante instrumento de análise e planejamento para gestores e demais profissionais envolvidos com as açôes de controle desse câncer. Além disto, estudos adicionais são imprescindíveis para a melhoria do conhecimento científico sobre este assunto e para a avaliação das estratégias adotadas para esta população.

\section{CONTRIBUIÇÕES}

Maria Asunción Solé Pla contribuiu na concepção e planejamento do artigo, na obtenção e/ou análise e interpretação dos dados e na redação e revisão crítica. Flávia Miranda Corrêa, Itamar Bento Claro, Marcos André Felix da Silva, Maria Beatriz Kneipp Dias e Paula Chagas Bortolon contribuíram na análise e interpretação dos dados e na redaçáo e revisão crítica.

\section{Declaraçáo de Conflito de Interesses: Nada a Declarar.}

\section{REFERÊNCIAS}

1. Leman RF, Espey D, Cobb N. Invasive cervical cancer among American Indian women in the Northern Plains, 1994-1998: incidence, mortality, and missed opportunities. Public Health Rep. 2005;120(3):283-7.

2. Elias B, Kliewer EV, Hall M, Demers AA, Turner D, Martens $\mathrm{P}$, et al. The burden of cancer risk in Canada's 
indigenous population: a comparative study of known risks in a Canadian region. Int J Gen Med. 2011;4:699-709.

3. National Aboriginal Health Organization. First Nations Centre. Cancer of the cervix in North American Indian women: a literature review. [Ottawa]: National Aboriginal Health Organization; [2006].

4. Instituto Brasileiro de Geografia e Estatística. Censo 2010 [Internet]. [citado 2012 abr 22]. Disponível em: http:// www.censo2010.ibge.gov.br/resultados_do_censo2010.php

5. Fundação Nacional de Saúde (Brasil). Política nacional de atenção à saúde dos povos indígenas. 2a ed. Brasília: FUNASA; 2002. 40 p.

6. Fundação Nacional de Saúde (Brasil). Departamento de Saúde Indígena. Relatório de gestão DESAI/CGASI 2010. Brasília: FUNASA; 2010. 91 p.

7. Brasil. Ministério da Saúde. Secretaria de Atenção à Saúde. Departamento de Atenção Básica. Controle dos cânceres do colo do útero e da mama. Brasília: Ministério da Saúde; 2006. 124 p. (Cadernos de Atenção Básica; n. 13); (Série A. Normas e manuais técnicos).

8. Albring L, Brentano JE, Vargas VRA. O câncer do colo do útero, o Papilomavírus Humano (HPV) e seus fatores de risco e as mulheres indígenas Guarani: estudo de revisão. Rev bras anal Clin. 2006;38(2):87-90.

9. Pereira ÉR. Prevenção do câncer do colo do útero em população feminina do Parque Indígena do Xingu, Mato Grosso [tese]. São Paulo: Universidade Federal de São Paulo; 2011.

10. Taborda WC, Ferreira SC, Rodrigues D, Stávale JN, Baruzzi RG. Rastreamento do câncer de colo uterino em índias do Parque Indígena do Xingu, Brasil central. Rev Panam Salud Públ / Pan Am J Public Health. 2000;7(2):92-6.

11. Mendes ECS. Prevalência de câncer de cérvice uterina e suas lesōes precursoras em população indígena do Estado do Pará. Rev para med. 2004;18(1):11-8.

12. Carvalho NS, Cho R, Flores LP. DST em populações indígenas no Brasil: análise crítica e revisão da literatura. DST j bras doenças sex transm. 2011;23(3):142-5.

13. International Agency for Research on Cancer. Cervix cancer screening. Lyon: IARC Press; 2005.302 p. (IARC handbooks of cancer prevention; vol. 10).
14. Instituto Nacional de Câncer (Brasil). Diretrizes brasileiras para o rastreamento do câncer do colo do útero. Rio de Janeiro: INCA; 2011. 104 p.

15. Instituto Nacional de Câncer (Brasil). Sistemas de Informação do Controle do Câncer de Mama (SISMAMA) e do Câncer do Colo do Útero (SISCOLO): manual gerencial. Rio de Janeiro: INCA; 2011.

16. Brasil. Ministério da Saúde. Departamento de Informática do SUS (DATASUS). Sistema de Informação do Câncer do Colo do Útero (SISCOLO) [Internet]. [citado 2012 abr 22]. Disponível em: http://w3.datasus.gov.br/siscam/ index.php

17. Instituto Nacional de Câncer (Brasil). Nomenclatura brasileira para laudos cervicais e condutas preconizadas: recomendações para profissionais de saúde. Rio de Janeiro: INCA; 2006.56 p.

18. Brasil. Ministério da Saúde. Departamento de Informática do SUS (DATASUS). Sistema de Informação de Mortalidade e Sistema de Informações Hospitalares do SUS [Internet]. [citado 2012 abr 2]. Disponível em: http://tabnet.datasus.gov.br

19. Instituto Brasileiro de Geografia e Estatística. Pesquisa nacional por amostra de domicílios: um panorama da saúde no Brasil: acesso e utilização dos serviços, condições de saúde e fatores de risco e proteção à saúde: 2008. Rio de Janeiro: IBGE; 2010.

20. Instituto Nacional de Câncer (Brasil). Plano de ação para redução da incidência e mortalidade por câncer do colo do útero: sumário executivo. Rio de Janeiro: INCA; 2010. 40 p.

21. Rodrigues DE, Escobar AL, Marques RC. Prevalência do câncer cérvico-uterino em mulheres indígenas Suruí e Cinta Larga, Rondônia, Brasil. Anais do XIV Seminário de Iniciação Científica; 2005 ago 1-5; Porto Velho, BR. Porto Velho: Editora da Universidade Federal de Rondônia; 2005.

22. Pereira JC, Ganassin FMH, Oliveira RD, Renovato RD, Watanabe EAMT. Morbidade por câncer de colo uterino em mulheres de reserva indígena no Mato Grosso do Sul. Cogitare enferm. 2011;16(1):127-33. 


\begin{abstract}
Introduction: There are 408.056 indigenous women in Brazil. This population is more vulnerable to disorders of great magnitude, including cervical cancer. However, there are few studies about this subject among indigenous women. Objective: To describe the profile of Pap smears performed in the Brazilian indigenous population compared to nonindigenous. Method: Frequency and proportions descriptive analysis of Pap smears performed among indigenous and non-indigenous women, by region and in the period 2008-2011, registered in the Brazilian Information System for Cervical cancer (SISCOLO). Results: Between 2008 and 2010, 8\% of Pap smears performed in Brazil registered the information of color/race and in 2011this proportion increased to $12.4 \%$. In indigenous and non-indigenous women, 11.964 and 3.738 .707 satisfactory tests were performed, respectively; $1 \%$ and $1.2 \%$ of tests were considered unsatisfactory; $74.9 \%$ and $77.2 \%$ of exams were performed between 25 and 64 years; $20 \%$ and $17.5 \%$ of tests were done in women under 25 years; $83.4 \%$ and $76.5 \%$ of exams were made within 1 to 2 years; $2.1 \%$ and $2.9 \%$ of tests were positive. The high-grade lesion/invasive cervical cancer ratio increased from 11.5 in 2009 to 16.1 in 2011 in non-indigenous women and from 1.7 to 5.0 among indigenous women. Conclusion: These data show how important this subject is for indigenous women, suggest lack of access to cervical screening for this group and highlight the need of improvement of color/race information in SISCOLO.

Key words: Uterine Cervical Neoplasms; Indigenous Population; Information Systems; Vaginal Smears
\end{abstract}

\title{
Resumen
}

Introducción: En Brasil hay 408.056 mujeres indígenas. Esta población es más vulnerable a las enfermedades de gran magnitud, incluyendo el cáncer del cuello del útero. Sin embargo, son pocos los estudios existentes acerca de esa cuestión entre las mujeres indígenas. Objetivo: Describir el perfil de pruebas de Papanicolaou realizadas en la población indígena de Brasil en comparación con las no-indígenas. Método: Análisis descriptiva de la frecuencia y de la proporción de las pruebas de Papanicolaou realizadas entre las mujeres indígenas y no indígenas registradas en el Sistema de Información del Cáncer Cervical (SISCOLO) por región de residencia para el período 2008 a 2011. Resultados: Entre 2008 y 2010, el 8\% de pruebas citopatológicas realizadas en el país registraron la información de raza / color, y en 2011 esta proporción se incremento al 12,4\%. En indígenas y no indígenas, respectivamente, se realizaron 11.964 y 3.738 .707 pruebas satisfactorias; se consideraron insatisfactorios el $1 \%$ y $1,2 \%$ de las pruebas; $74,9 \%$ y el $77,2 \%$ de las pruebas se realizaron en la franja de edad de 25 a 64 años; $20 \%$ y el 17,5\% de las pruebas se realizaron antes de los 25 ańos; $83,4 \%$ y 76,5\% de las pruebas se realizaron en el periodo de 1 a 2 ańos; $2,1 \%$ y 2,9\% de pruebas se presentaron anormales. La razón lesión de alto grado / cáncer invasor del cuello del útero pasó del 11,5 en 2009 a 16,1 en 2011 en las no indígenas, y 1,7 para 5,0 entre las mujeres indígenas. Conclusión: Los datos evidencian la importancia del tema entre las mujeres indígenas, sugieren la dificultad de acceso a las pruebas del cuello del útero para este grupo y destacan la necesidad de mejorar la información de color/raza en el sistema de información, SISCOLO.

Palabras clave: Neoplasias del Cuello Uterino; Población Indígena; Sistemas de Información; Frotis Vaginal 